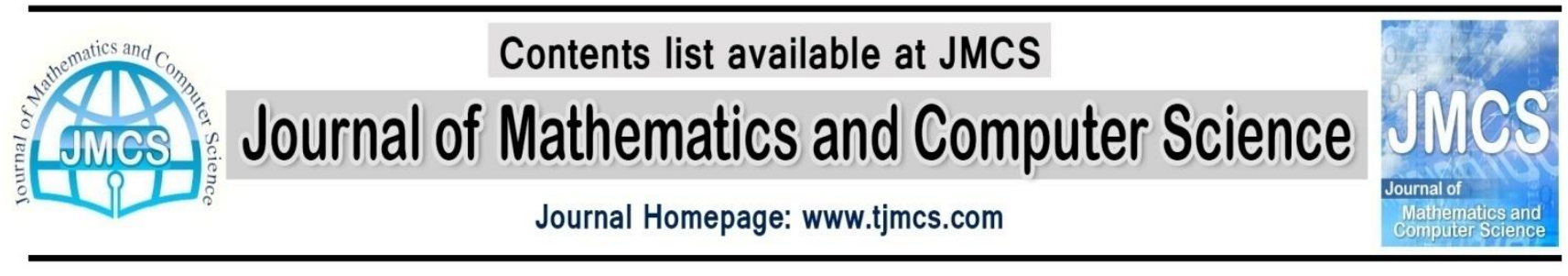

\title{
Numerical Solution of Black-Scholes Equation Using Bernstein Multi-Scaling Functions
}

\author{
M. Moradipour ${ }^{1}$, S. A. Yousefi, ${ }^{1, *}$ \\ ${ }^{1}$ Department of Mathematics, Shahid Beheshti University, G.C., Tehran, Iran \\ s-yousefi@sbu.ac.ir \\ Article history: \\ Received March 2015 \\ Accepted July 2015 \\ Available online July 2015
}

\section{Abstract}

A numerical method for solving Black-Scholes equation is presented. The method is based upon Bernstein multi-scaling basis approximations. The properties of Bernstein multi-scaling functions are first presented. These properties together with the forward Euler and Ritz-Galerkin method are then utilized to reduce the Black-Scholes equation to the solution of algebraic equations. Illustrative example is included to demonstrate the validity and applicability of the new technique.

Keywords: Bernstein polynomial, Bernstein multi-scaling functions, Black-Scholes equation, Euler method, Ritz-Galerkin method.

\section{Introduction}

Finance is one of the most rapidly changing and fastest growing areas in the corporate business world. Because of this rapid change, modern financial instruments have become extremely complex. Options are one of the most important types of financial instruments, for a detailed discussion of options the reader is referred to [2], [3]. In 1973, a major breakthrough occurred in the field of finance when researchers Fischer Black and Myron Scholes developed a model for pricing options using continuous-time mathematics. Black and Scholes transformed the option pricing problem into the task of solving a (parabolic) partial differential equation (PDE) with a final condition [1]. European option prices (in the put case) under Black-Scholes model satisfy the well-known Black-Scholes partial differential equation:

$$
\begin{aligned}
& u_{t}(x, t)+\frac{1}{2} \sigma^{2} x^{2} u_{x x}(x, t)+r x u_{x}(x, t)-r u(x, t)=0, \quad(x, t) \in[0, \infty) \times[0, T], \\
& u(x, T)=\max (K-x, 0), \\
& u(0, t)=K e^{-r(T-t)}, \quad \lim _{x \rightarrow \infty} u(x, t)=0 .
\end{aligned}
$$

Where the strike price $K$, the risk free interest rate $r$, the volatility $\sigma$, and the expiry date $T$, are all positive constants. We can transform the Black-Scholes equation (1) to the heat equation by change of variables, having found the closed-form solution to the heat equation, it is possible to transform it back to find the corresponding solution of the Black-Scholes equation. See [2] for details. Using numerical methods, one 
can approximate solutions of Black-Scholes differential equation. Some numerical methods such as finite difference, finite element and binomial ones were used for this purpose $[4,5,6,7,8,9,10]$. In this article we are aimed to use Bernstein Ritz-Galerkin method for approximate solutions of Black-Scholes partial differential equation (1).

\section{Properties of Bernstein polynomials}

The Bernstein polynomials of $\mathrm{m}$-th degree are defined on the interval $[\mathrm{a}, \mathrm{b}]$ as $[11,12,13]$

where

$$
B_{i, m}(x)=\left(\begin{array}{c}
m \\
i
\end{array}\right) \frac{(x-a)^{i}(b-x)^{m-i}}{(b-a)^{m}}, \quad 0 \leq i \leq m
$$

$$
\left(\begin{array}{c}
m \\
i
\end{array}\right)=\frac{m !}{i !(m-i) !}
$$

These Bernstein polynomials form a basis on $[\mathrm{a}, \mathrm{b}]$. There are $\mathrm{m}+1$ polynomials of degrees $\mathrm{m}$. For convenience, we set $B_{i, m}(x)=0$ if $i<0$ or $i>m$. A recursive definition can also be used to generate the Bernstein polynomials over $[a, b]$ so that the $i$-th $m$-th degree Bernstein polynomials can be written

$$
B_{i, m}(x)=\frac{(b-x)}{b-a} B_{i, m-1}(x)+\frac{(x-a)}{b-a} B_{i-1, m-1}(x) .
$$

It can readily be shown that each of the Bernstein polynomials is positive and the sum of all the Bernstein polynomials is unity for all real $x \in[a, b]$, i.e. $\sum_{i=0}^{m} B_{i, m}(x)=1$. It is easy to show that any given polynomial of degree $\mathrm{m}$ can be expanded in terms of these basis functions.

\section{Properties of Bernstein multi-scaling functions}

Bernstein multi-scaling functions $\psi_{i, n}(t)=B_{i, m}(k t-n), \quad i=0,1, \ldots, m$, have four arguments; translation argument $n=0,1, \ldots, k-1$, argument $k>1$ can assume any positive integer, $m$ is the order of Bernstein polynomial on $[0,1]$ and $t$ is the normalized time. They are defined on the interval $[0,1)$ as

$$
\psi_{i, t}(t)=\left\{\begin{array}{lc}
B_{i, m}(k t-n), & \frac{n}{k} \leq t \leq \frac{n+1}{k} \\
0, & \text { otherwise }
\end{array}\right.
$$

\subsection{Function Approximation}

Suppose that $\mathrm{H}=\mathrm{L}^{2}[0,1]$ and $\left\{\psi_{i, n}(t)\right\}_{i=0, n=0}^{m, k-1} \subset H$ be the set of multi scaling-functions of $m$-th degree and

$$
Y=\operatorname{Span}\left\{\psi_{i, n}(t) ; i=0,1, \ldots, m, n=0,1, \ldots, k-1\right\},
$$

and $f$ be an arbitrary element in $H$. Since $Y$ is a finite dimensional vector space, $f$ has the unique best approximation out of $Y$ such as $\mathrm{y}_{0} \in \mathrm{Y}$ that is

where $\|f\|_{2}=\sqrt{\langle f, f\rangle}$.

$$
\exists y_{0} \in Y ; \quad \forall y \in Y \quad\left\|f-y_{0}\right\|_{2} \leq\|f-y\|_{2},
$$


Since $y_{0} \in Y$ there exist the unique coefficients $c_{i, n}$ such that

where

$$
f \simeq y_{0}=\sum_{n=0}^{k-1} \sum_{i=0}^{m} c_{i, n} \psi_{i, n}=\boldsymbol{C}^{\top} \boldsymbol{\phi},
$$

$$
\begin{gathered}
\boldsymbol{\phi}^{\top}=\left[\psi_{0,0}, \psi_{1,0}, \ldots, \psi_{m-1,0}, \psi_{m, 0}, \ldots, \psi_{0, k-1}, \psi_{1, k-1}, \ldots, \psi_{m-1, k-1}, \psi_{m, k-1}\right], \\
\boldsymbol{C}^{\top}=\left[c_{0,0}, c_{1,0}, \ldots, c_{m-1,0}, c_{m, 0}, \ldots, c_{0, k-1}, c_{1, k-1}, \ldots, c_{m-1, k-1}, c_{m, k-1}\right]
\end{gathered}
$$

and $\boldsymbol{C}^{\top}$ can be obtained by

where

$$
\boldsymbol{C}^{\top}\langle\boldsymbol{\phi}, \boldsymbol{\phi}\rangle=\langle f, \boldsymbol{\phi}\rangle,
$$

$$
\langle f, \boldsymbol{\phi}\rangle=\int_{0}^{1} f(x) \boldsymbol{\phi}(x)^{\top},
$$

and $\langle\boldsymbol{\phi}, \boldsymbol{\phi}\rangle$ is a $k(m+1) \times k(m+1)$ matrix which is said dual operational matrix of $\boldsymbol{\phi}$ denoted by $\overline{\boldsymbol{Q}}$ and will be obtained in the following.

And then

$$
\overline{\mathbf{Q}}=\langle\boldsymbol{\phi}, \boldsymbol{\phi}\rangle=\int_{0}^{1} \phi(x) \phi(x)^{\top},
$$

$$
\boldsymbol{C}^{\top}=\left(\int_{0}^{1} f(x) \boldsymbol{\phi}(x)^{\top}\right)(\overline{\mathbf{Q}})^{-1}
$$

In the following lemma we present an upper bound for the error approximation.

Lemma 1. Suppose that the function $g:[0,1) \rightarrow \mathbb{R}$ is $m+1$ times continuously differentiable, $g \in C^{m+1}\left[t_{0}, t_{f}\right]$ and

$$
Y=\operatorname{Span}\left\{\psi_{i, n}(t) ; \quad i=0,1, \ldots m, \quad n=0,1, \ldots, k-1\right\}
$$

If $\boldsymbol{C}^{\top} \boldsymbol{\phi}$ is the best approximation $g$ out of $Y$, then the mean error bounded is presented as follows:

$$
\left\|g-\boldsymbol{C}^{\top} \boldsymbol{\phi}\right\|_{2} \leq \frac{M}{(m+1) ! k^{m+1} \sqrt{2 m+3}},
$$

where $M=\max _{x \in\left[\mathrm{t}_{0}, \mathrm{t}_{\mathrm{f}}\right]}\left|g^{m+1}(x)\right|$.

Proof. We consider the Taylor polynomial of order $m$ for function $g$ on $\left[\frac{n}{k}, \frac{n+1}{k}\right)$.

$$
y_{n}(x)=g\left(\frac{n}{k}\right)+g^{\prime}\left(\frac{n}{k}\right)\left(x-\frac{n}{k}\right)+\cdots+g^{(m)}\left(\frac{n}{k}\right) \frac{\left(x-\frac{n}{k}\right)^{m}}{m !},
$$

for $n=0,1, \ldots, k-1$ which we know

$$
\left|g(x)-y_{n}(x)\right| \leq\left|g^{m+1}(\eta)\right| \frac{\left(x-\frac{n}{k}\right)^{m+1}}{(m+1) !},
$$

where $\eta \in\left(\frac{n}{k}, \frac{n}{k+1}\right)$. Since $\boldsymbol{C}^{\top} \boldsymbol{\phi}$ is the best approximation of $g$ out of $Y, y_{n} \in Y$ and using (4) we have

$$
\left\|g-\boldsymbol{C}^{\top} \boldsymbol{\phi}\right\|_{2}^{2}=\int_{0}^{1}\left|g(x)-\boldsymbol{C}^{\top} \boldsymbol{\phi}(x)\right|^{2} d x=\sum_{n=0}^{k} \int_{\frac{n}{k}}^{\frac{n+1}{k}}\left|g(x)-\boldsymbol{C}^{\top} \boldsymbol{\phi}(x)\right|^{2} d x
$$




$$
\begin{aligned}
& \leq \sum_{n=0}^{k-1} \int_{\frac{n}{k}}^{\frac{n+1}{k}}\left|g(x)-y_{n}(x)\right|^{2} d x \leq \sum_{n=0}^{k-1} \int_{\frac{n}{k}}^{\frac{n+1}{k}}\left[g^{(m+1)}(\eta) \frac{\left(x-\frac{n}{k}\right)^{m+1}}{(m+1) !}\right]^{2} d x \\
& \leq \frac{M^{2}}{(m+1) !} \sum_{n=0}^{k-1} \int_{\frac{n}{k}}^{\frac{n+1}{k}}\left(x-\frac{n}{k}\right)^{2 m+2} d x=\frac{M^{2}}{[(m+1) !]^{2} k^{2 m+2}(2 m+3)}
\end{aligned}
$$

and by taking square root we have the above bound.

Using the Bernstein scaling functions basis, we will have two degrees of freedom which increase the accuracy of the approximation. One of these parameters is the argument $k$ and another one is $m$ which corresponds to the degree of Bernstein polynomials in every subinterval $\left[\frac{n}{k}, \frac{n+1}{k}\right)$. As can be seen in the above lemma, the upper bound of the error depends on $\frac{1}{(m+1) ! k^{m+1} \sqrt{2 m+3}}$ which shows that the error reduces to zero very fast as $m$ and $k$ increase. This is one of the advantages of the Bernstein scaling approximation.

\section{Bernstein Ritz Galerkin method}

By change of variable $t \rightarrow T-t$ we can rewrite equation (1) as

$$
\begin{aligned}
& u_{t}(x, t)-\mathcal{L}(u(x, t))=0, \quad(x, t) \in[0, \infty) \times[0, T], \\
& u(0, t)=K e^{-r t}, \quad \lim _{x \rightarrow \infty} u(x, t)=0 .
\end{aligned}
$$

Where $\mathcal{L}$ is a linear partial differential operator introduced by

$$
\mathcal{L}(\phi)=\frac{1}{2} \sigma^{2} x^{2} \frac{\partial^{2} \phi}{\partial x^{2}}+r x \frac{\partial \phi}{\partial x}-r \phi .
$$

The semi-infinite domain in the space direction is replaced by an interval $[0, L]$ for a sufficiently large $L$. Given the discretization of the time interval $[0, T]$

$$
0=t_{0}<t_{1}<\cdots<t_{N}=T,
$$

with time step size $t=t_{n+1}-t_{n}$, the forward Euler method for equation (5) is

$$
u\left(x, t_{n+1}\right)-\delta t \mathcal{L}\left(u\left(x, t_{n+1}\right)\right)=u\left(x, t_{n}\right),
$$

so for each time step, we have an ordinary differential equation with two boundary conditions. In equation (6) $u\left(x, t_{n+1}\right)$ is unknown and $u\left(x, t_{n}\right)$ in the right hand side is obtain from initial condition of PDE.

Let $\left\{\psi_{i, n}(t)\right\}_{i=0, n=0}^{m, k-1}$ be the Bernestein scaling functions defined as (2) so the total number of this functions is $M=k(m+1)$ We can rewrite these functions as $\phi_{1}(x), \phi_{2}(x), \ldots, \phi_{M}(x)$ by changing indices, where

$$
\psi_{i n}(x)=\phi_{i+n(m+1)+1}(x):=\phi_{j}(x), j=1,2, \ldots, M .
$$

The approximation of the unknown function $u\left(x, t_{n+1}\right)$ may be written as 


$$
u\left(x, t_{n+1}\right)=\sum_{j=1}^{M} c_{j} \phi_{j}(x)=\boldsymbol{C}^{\top} \boldsymbol{\phi}
$$

Where $\mathbf{C}=\left[\mathrm{c}_{1}, \mathrm{c}_{2}, \ldots, \mathrm{c}_{\mathrm{M}}\right]^{\top}$ is the vector of unknown coefficient that must be found and $\boldsymbol{\phi}=\left[\phi_{1}(x), \phi_{2}(x), \ldots \phi_{M}(x)\right]^{\top}$ is the vector of basis functions. Substituting equation (7) into equation (6) yields

$$
\sum_{j=1}^{M} c_{j}\left(\phi_{j}(x)-\delta t \mathcal{L}\left(\phi_{j}(x)\right)\right)=u\left(x, t_{n}\right)
$$

Taking the inner product from both sides of $(8)$ with $\phi_{i}(x), i=1,2, \ldots, M$ yields

$$
\sum_{j=1}^{M} c_{j}\left\langle\left(\phi_{j}(x)-\delta t \mathcal{L}\left(\phi_{j}(x)\right)\right), \phi_{i}(x)\right\rangle=\left\langle u\left(x, t_{n}\right), \phi_{i}(x)\right\rangle, \quad i=2,3, \ldots, M-1 .
$$

From boundary conditions we have

$$
\sum_{j=1}^{M} c_{j} \phi_{j}(0)=K e^{-r t_{n+1}}, \quad \sum_{j=1}^{M} c_{j} \phi_{j}(L)=0 .
$$

The linear algebraic equations (9) and (10) can be written as the matrix form

$$
A C=b
$$

Where the matrix $\boldsymbol{A}$ has entries

$$
\begin{aligned}
& \boldsymbol{A}_{i j}=\int_{0}^{L}\left(\phi_{j}(x)-\delta t \mathcal{L}\left(\phi_{j}(x)\right)\right) \phi_{i}(x) d x, \quad j=1,2, \ldots, M \quad i=2,3, \ldots, M-1, \\
& \boldsymbol{A}_{1 j}=\phi_{j}(0), \quad j=1,2, \ldots, M \\
& \boldsymbol{A}_{M j}=\phi_{j}(L), \quad j=1,2, \ldots, M
\end{aligned}
$$

and the elements of right hand side vector $\boldsymbol{b}$ are

$$
\begin{aligned}
& \boldsymbol{b}_{i}=\int_{0}^{L} u\left(x, t_{n}\right) \phi_{i}(x) d x, \quad i=2,3, \ldots, M-1, \\
& \boldsymbol{b}_{1}=K e^{-r t_{n+1}} \\
& \boldsymbol{b}_{M}=0 .
\end{aligned}
$$

We have to solve the system of linear equation (11) at $n$-th time step to find the solution $u\left(x, t_{n}\right)$ for $n=2,3, \ldots, N$. We would notice that the coefficients matrix $\boldsymbol{A}$ is unchanged in time steps.

\section{Numerical results}

To illustrate the description above and to test the Bernstein Ritz-Galerkin (BRG) method developed here for solving the Black-Scholes equation, we give a numerical example.

Consider (5) with parameters

$$
K=10, \quad T=0.5, \quad r=0.5, \quad \sigma=0.25 \text {. }
$$


The analytical solution satisfying the $\operatorname{PDE}\left({ }^{\circ}\right)$ with the given initial and boundary conditions is [2]

Where

$$
u(x, t)=K e^{-r t} \mathcal{N}(-d 2)-x \mathcal{N}(-d 1),
$$

$$
\mathcal{N}(y)=\frac{1}{\sqrt{2 \pi}} \int_{-\infty}^{y} e^{-\frac{u^{2}}{2}} d u
$$

Is the cumulative distribution function of standard normal distribution and

$$
\begin{aligned}
& d_{1}=\frac{\ln \left(\frac{x}{K}\right)+\left(r+\frac{\sigma^{2}}{2} t\right)}{\sigma \sqrt{t}}, \\
& d_{2}=\frac{\ln \left(\frac{x}{K}\right)+\left(r-\frac{\sigma^{2}}{2} t\right)}{\sigma \sqrt{t}} .
\end{aligned}
$$

We use the BRG method with $M=30, k=3$ and $N=101$ temporal levels to compute $\mathrm{u}(\mathrm{x}, \mathrm{t})$. Financially the value of $u(x, T)$ in equation (5) is important because it shows the price of option at present time. The results are presented in table 1 . Figure 1 shows the solution of PDE in the entire of domain. Figures 2 and 3 present the solution and the absolute error at time $t=T$ versus the parameter $x$ respectively.

Table 1: Comparison between BRG and exact solution to $u(x, t)$ at the time $t=T$ for various values of $x$.

\begin{tabular}{crrrrc}
\hline $\boldsymbol{x}$ & $\begin{array}{c}\text { Exact } \\
\text { solutions }\end{array}$ & $\begin{array}{c}\text { BRG } \\
\text { solutions }\end{array}$ & $\boldsymbol{x}$ & $\begin{array}{c}\text { Exact } \\
\text { solutions }\end{array}$ & $\begin{array}{c}\text { BRG } \\
\text { solutions }\end{array}$ \\
\hline 1 & 8.7530 & 8.7531 & 11 & 0.2697 & 0.2690 \\
2 & 7.7530 & 7.7531 & 12 & 0.1131 & 0.1130 \\
3 & 6.7530 & 6.7531 & 13 & 0.0434 & 0.0436 \\
4 & 5.7530 & 5.7531 & 14 & 0.0154 & 0.0157 \\
5 & 4.7531 & 4.7531 & 15 & 0.0052 & 0.0054 \\
6 & 3.7543 & 3.7544 & 16 & 0.0016 & 0.0017 \\
7 & 2.7702 & 2.7706 & 17 & 0.0005 & 0.0005 \\
8 & 1.8556 & 1.8560 & 18 & 0.0001 & 0.0001 \\
9 & 1.1038 & 1.1034 & 19 & 0.0000 & 0.0000 \\
10 & 0.5791 & 0.5782 & 20 & 0.0000 & 0.0000 \\
\hline
\end{tabular}

From Table 1, it can be seen that the BRG method provides an accurate approximation for pricing European options. We observe that the accuracy grows as the number of basis increases gradually, then the solution can be computed with a small error in a small computer time. 


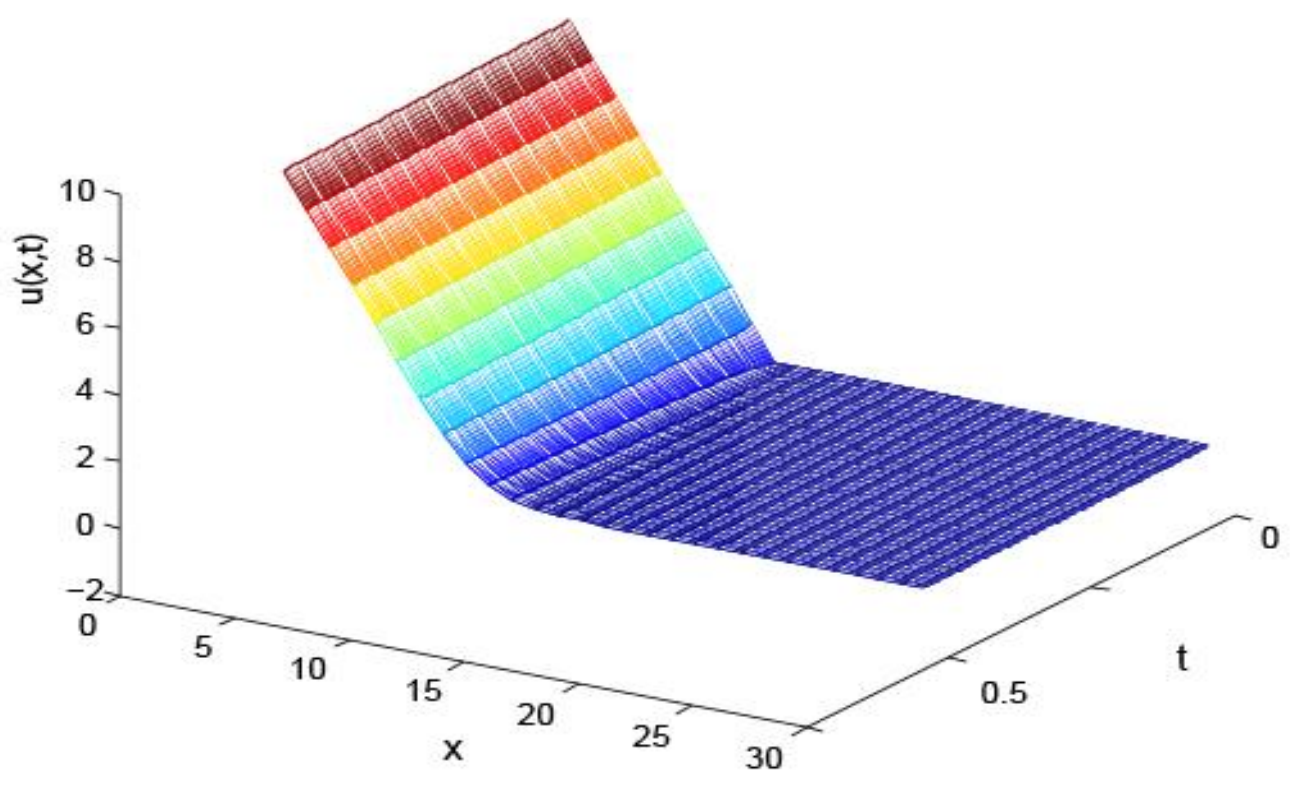

Figure 1: Surface plot of the numerical solution $u(x, t)$ at the entire of domain $[0, L] \times[0, T]$.

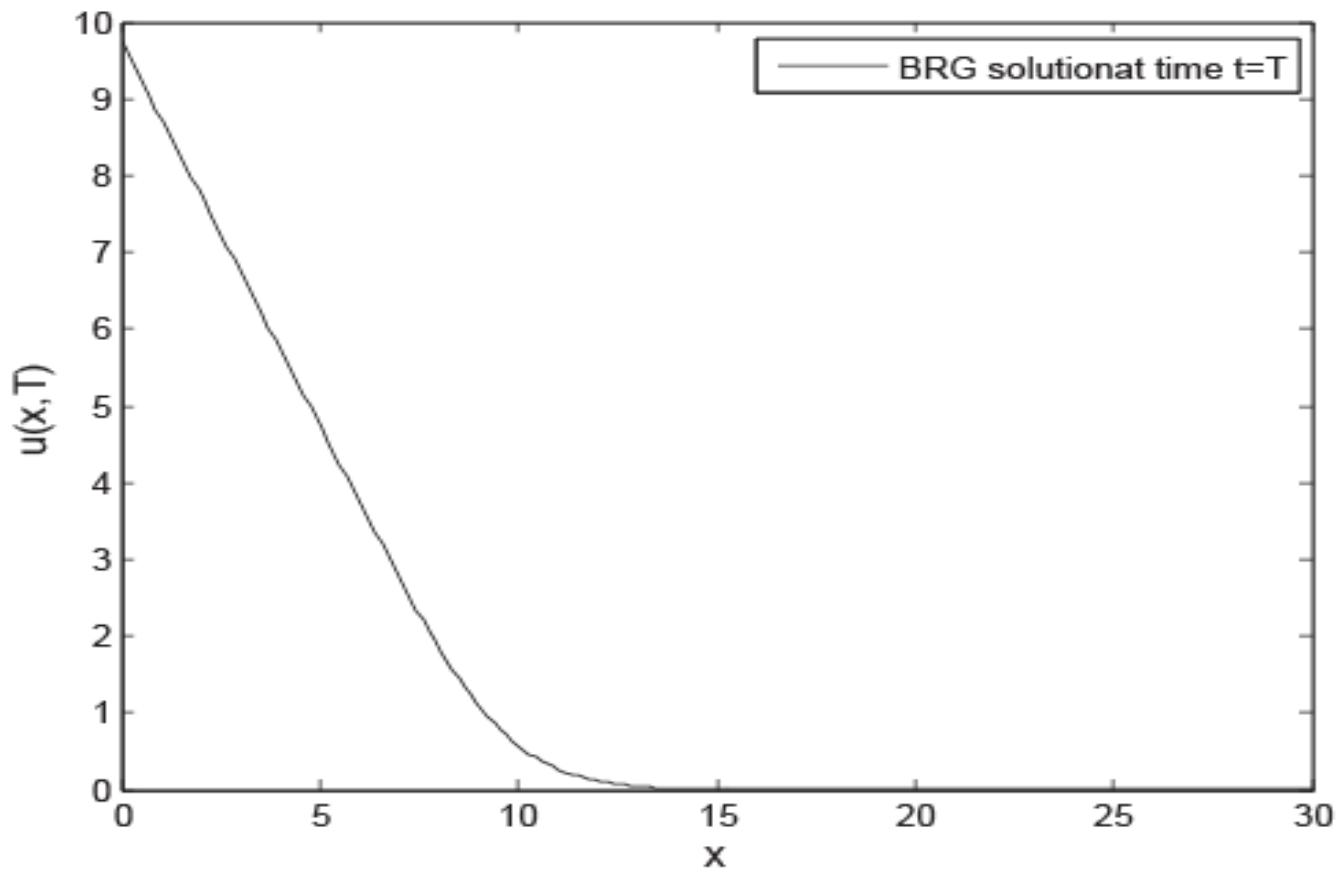

Figure 2: Curve plot of the numerical solution $u(x, t)$ at time $t=T$ by using $\mathrm{M}=30, k=3$ and $N=101$ temporal levels. 


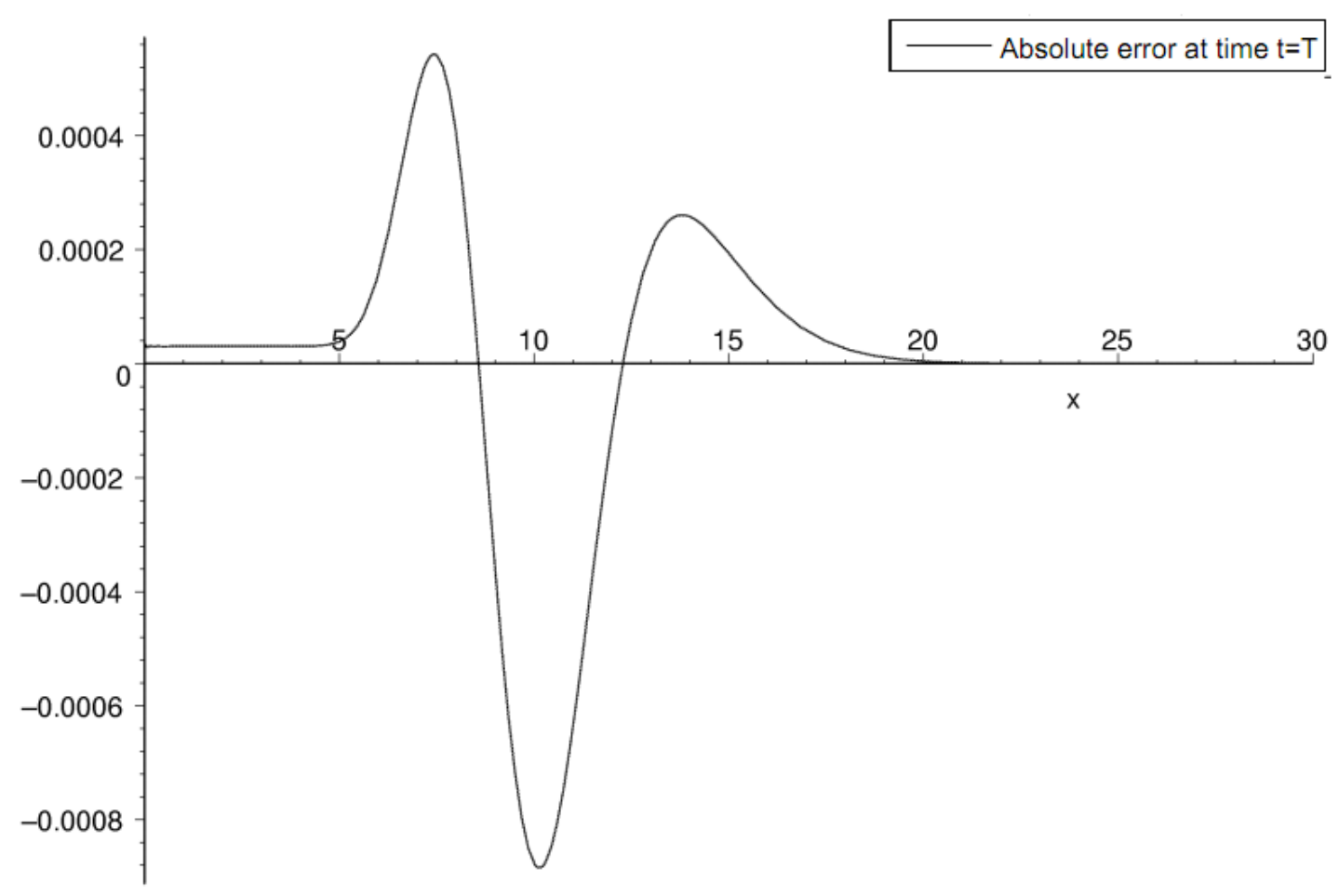

Figure 3: Absolute error of the BRG solution $u(x, t)$ at time $t=T$ by using $\mathrm{M}=30, k=3$ and $N=101$ temporal levels.

\section{Conclusions}

In this article the Bernstein multi-scaling approximation in forward Euler and Ritz-Galerkin method was used successfully for solving the Black-Scholes equation. Bernstein multi-scaling functions as basis have different resolution capabilities for expanding functions and therefore by increasing dilation parameter $k$ we get local approximation and this is good for equations that have non-smooth solution. Illustrative example is included to demonstrate the validity and applicability of the new technique.

\section{References}

[1] F. Black, M. Scholes, "The pricing of options and corporate liabilities", Journal of Political Economy, (1973), 637-659.

[2] J. C. Hull, "Options, futures, and other derivatives", Pearson Prentice Hall, Upper Saddle River, NJ, 6.ed, (2006).

[3] P. Wilmott, "Introduces quantitative finance", John Wiley \& Sons, (2007).

[4] Y. Achdu, O. Pironneau, "Computational methods for option pricing”, SIAM, (2005).

[5] D. J. Duffy, "Finite Difference Methods in Financial Engineering", John Wiley \& Sons, (2006).

[6] J. C. Cox, "Option pricing: a simplified approach", Journal of Financial Economics 7 (1979), 229263. 
[7] M. Dehghan, S. Pourghanbar, "Solution of the Black-Scholes equation for pricing of barrier option”, Z. Naturforsch., A, Vol. 66a (2011), 289-296.

[8] J. Ankudinova, M. Ehrhardt, "On the numerical solution of nonlinear Black-Scholes equations", Computers and Mathematics with Applications 56 (2008), 799-812.

[9] R. Company, E. Navarro, J. R. Pintos, E. Ponsoda, "Numerical solution of linear and nonlinear Black-Scholes option pricing equations", Computers and Mathematics with Applications 56 (2008), 813-821.

[10] M. Chawla, M. Al-zanaidi, D. Evans, "Generalized Trapezoidal Formulas for the Black-Scholes Equation of Option Pricing", International Journal of Computer Mathematics Vol. 80, No. 12, (2003), 1521-1526.

[11] M. Idrees Bhatti, P. Bracken, "Solutions of differential equations in a Bernstein polynomial basis", J. Comput. Appl. Math. 205 (2007), 272-280.

[12] S. A. Yousefi, M. Behroozifar, "Operational matrices of Bernstein polynomials and their applications", International Journal of Systems Science, Vol. 41, No. 6, (2010), 709-716.

[13] S. A. Yousefi, "B-polynomial multiwavelets approach for the solution of Abel's integral equation”, International Journal of Computer Mathematics, Vol. 87, No. 2, (2010), 310-316. 